PROCEEDINGS OF THE

AMERICAN MATHEMATICAL SOCIETY

Volume 132, Number 11, Pages 3275-3281

S 0002-9939(04)07446-5

Article electronically published on June 17, 2004

\title{
NESTS WITH THE PARTIAL FACTORIZATION PROPERTY
}

\author{
GUOXING JI AND XIUHONG SUN
}

(Communicated by Joseph A. Ball)

\begin{abstract}
It is proved that a nest $\mathcal{N}$ on a separable complex Hilbert space $\mathcal{H}$ has the left (resp. right) partial factorization property, which means that for every invertible operator $T$ from $\mathcal{H}$ onto a Hilbert space $\mathcal{K}$ there exists an isometry (resp. a coisometry) $U$ from $\mathcal{H}$ into $\mathcal{K}$ such that both $U^{*} T$ and $T^{-1} U$ are in the associated nest algebra $A l g \mathcal{N}$ if and only if it is atomic (resp. countable).
\end{abstract}

\section{INTRODUCTION}

Factorization problems in operator algebras have been studied for several decades. One of these problems is the factorization problem relative to a nest algebra (cf. 11, 2, 3, 4, 5, 7, 8, 9]). Let $\mathcal{H}$, and $\mathcal{K}$ be separable complex Hilbert spaces, and let $\mathcal{B}(\mathcal{H}, \mathcal{K})$ be the set of all bounded operators from $\mathcal{H}$ into $\mathcal{K} . \mathcal{B}(\mathcal{H})$ denotes the algebra of all bounded linear operators on $\mathcal{H}$. A nest on $\mathcal{H}$ is a family of closed subspaces of $\mathcal{H}$ totally ordered by inclusion that is complete in the sense that it contains (0) and $\mathcal{H}$ and contains the closed linear span and intersection of every subfamily. The associated nest algebra is $\operatorname{Alg} \mathcal{N}=\{A \in \mathcal{B}(\mathcal{H}): A N \subseteq N, N \in \mathcal{N}\}$. The core $\mathfrak{E}_{\mathcal{N}}$ is the von Neumann algebra generated by the projections onto the members of $\mathcal{N}$, and the diagonal is $\mathcal{D}_{\mathcal{N}}=(A \lg \mathcal{N}) \cap(A \lg \mathcal{N})^{*}=\left(\mathfrak{E}_{\mathcal{N}}\right)^{\prime}$. For $N \in \mathcal{N}$, define

$$
N_{-}=\vee\left\{N^{\prime} \in \mathcal{N}: N^{\prime}<N\right\} .
$$

The subspaces $N \ominus N_{-}$are called the atoms of $\mathcal{N}$. If the atoms of $\mathcal{N}$ span $\mathcal{H}, \mathcal{N}$ is called atomic. If there are no atoms, $\mathcal{N}$ is called continuous. If $\mathfrak{E}_{\mathcal{N}}$ is a maximal abelian von Neumann algebra, we say $\mathcal{N}$ is of multiplicity one (free).

Let $T$ be an invertible operator in $\mathcal{B}(\mathcal{H}, \mathcal{K})$. We say that $T$ has a factorization relative to $\mathcal{N}$ if there exists a unitary operator $U$ in $\mathcal{B}(\mathcal{H}, \mathcal{K})$ and an invertible operator $A \in A l g \mathcal{N} \cap(A l g \mathcal{N})^{-1}$ such that $T=U A$. If every invertible operator $T$ in $\mathcal{B}(\mathcal{H}, \mathcal{K})$ has such a factorization, we say that $\mathcal{N}$ has the factorization property. It is a well-known result given by Larson 7 that $\mathcal{N}$ has the factorization property if and only if $\mathcal{N}$ is countable. Thus any uncountable nest does not have this property.

Received by the editors April 30, 2003 and, in revised form, July 11, 2003.

2000 Mathematics Subject Classification. Primary 47L35.

Key words and phrases. Nest, nest algebra, left (resp. right) partial factorization, factorization. This research was supported in part by the National Natural Science Foundation of China (No. 10071047), the Excellent Young Teachers Program of MOE, P.R.C. and the China Scholarship Council. 
We note that $T$ having a factorization relative to $\mathcal{N}$ is equivalent to the existence of a unitary operator $U$ in $\mathcal{B}(\mathcal{H}, \mathcal{K})$ such that both $U^{*} T$ and $T^{-1} U$ are in $A l g \mathcal{N}$. Then Pitts introduced the general notion of partial factorization in [8]. He gave a definition for a general subalgebra of a von Neumann algebra. We are concerned with nest algebras; so we restrict here only to nests.

Let $T$ be an invertible operator in $\mathcal{B}(\mathcal{H}, \mathcal{K})$. We say that $T$ has a left (resp. right) partial factorization relative to $\mathcal{N}$ if there exists an isometry (resp. a coisometry) $U$ in $\mathcal{B}(\mathcal{H}, \mathcal{K})$ such that both $U^{*} T$ and $T^{-1} U$ are in $A l g \mathcal{N}$. It is known that $T$ has a factorization relative to $\mathcal{N}$ if and only if $T$ has both a left and a right partial one by Proposition 4.4 in [8]. If every invertible operator $T$ in $\mathcal{B}(\mathcal{H}, \mathcal{K})$ has such a left (resp. right) partial factorization, we say that $\mathcal{N}$ has the left (resp. right) partial factorization property. Pitts proved that an injective nest in a von Neumann algebra has the left partial factorization property in [8], and some related results are obtained in 6] by $\mathrm{Ji}$ and Saito. In this note, we consider those nests with the left (resp. right) partial factorization property in $\mathcal{B}(\mathcal{H})$. We prove that a nest $\mathcal{N}$ has the left partial factorization property if and only if it is atomic; $\mathcal{N}$ has the right partial factorization property if and only if it has the factorization property, that is, if and only if it is countable.

We will denote by $I$ the identity operator on a Hilbert space. For a closed subspace $M$ of $\mathcal{H}$, we denote by $P_{M}$ the orthogonal projection from $\mathcal{H}$ onto $M$. Let $T$ be an invertible operator in $\mathcal{B}(\mathcal{H}, \mathcal{K})$, and put $T \mathcal{N}=\{T N: N \in \mathcal{N}\}$.

\section{Partial FACtorization}

Let $\mathcal{N}$ and $\mathcal{M}$ be two nests acting on separable Hilbert spaces $\mathcal{H}$ and $\mathcal{K}$ respectively, and let $\theta$ be an order isomorphism from $\mathcal{N}$ onto $\mathcal{M}$. We say $\theta$ is unitary implementing if there is a unitary operator $U$ from $\mathcal{H}$ onto $\mathcal{K}$ such that $\theta(N)=U N$ for all $N \in \mathcal{N}$.

Proposition 1. Let $\mathcal{N}$ be a nest, and let $T$ be an invertible operator in $\mathcal{B}(\mathcal{H}, \mathcal{K})$.

(1) $T$ has a left partial factorization relative to $\mathcal{N}$ if and only if there is a projection $E$ in $\mathfrak{D}_{T \mathcal{N}}$ such that the map $N \rightarrow E(T N)$ is unitary implementing.

(2) $T$ has a right partial factorization relative to $\mathcal{N}$ if and only if there is a projection $E$ in $\mathfrak{D}_{\mathcal{N}}$ such that the map $E(N) \rightarrow T N$ is unitary implementing.

Proof. (1) $(\Longrightarrow)$ Let $U \in \mathcal{B}(\mathcal{H})$ be an isometry so that $U^{*} T, T^{-1} U \in A \lg \mathcal{N}$. Then $T^{-1} U U^{*} T \in A l g \mathcal{N}$. It follows that $E=U U^{*} \in A l g(T \mathcal{N})$ is a projection and therefore is in its diagonal $\mathfrak{D}_{T \mathcal{N}}$. Clearly, $U$ is a unitary operator from $\mathcal{H}$ onto $E \mathcal{H}$. For every $N \in \mathcal{H}$, we have $U^{*} T N \subseteq N$ and $T^{-1} U N \subseteq N$. Then $U N \subseteq T N$, which implies that $N \subseteq U^{*} T N \subseteq N$ since $U$ is an isometry. Thus $U^{*} T N=N$ and $U N=U U^{*} T N=E(T N)$. Then the map $N \rightarrow E(T N)$ is unitary implementing.

$(\Longleftarrow)$ Let $E \in \mathfrak{D}_{T \mathcal{N}}$ be a projection such that the map $N \rightarrow E(T N)$ is unitary implementing. Let $U$ be a unitary operator from $\mathcal{H}$ onto $E \mathcal{H}$ so that $U N=E(T N)$ for every $N \in \mathcal{N}$. Then $U$ may be regarded as an isometry on $\mathcal{H}$ such that $U U^{*}=E$. Thus we have $U N=E(T N)=U U^{*} T N$, which implies that $U^{*} T N=N$ for every $N \in \mathcal{N}$. Then $U^{*} T \in A \lg \mathcal{N}$. On the other hand, since $E=U U^{*} \in \operatorname{Alg}(T \mathcal{N})$, it follows that $T^{-1} U U^{*} T \in A \lg \mathcal{N}$. Hence $T^{-1} U N=T^{-1} U U^{*} T N \subseteq N$. That is, $T^{-1} U \in A l g \mathcal{N}$. Then $T$ has a left partial factorization relative to $\mathcal{N}$.

The proof of (2) is similar. The proof is complete. 
We now consider nests with the left partial factorization property.

Lemma 1. Let $\mathcal{N}$ be a continuous nest. Then $\mathcal{N}$ does not have the left partial factorization property.

Proof. Assume that $\mathcal{N}$ is continuous. By Lemma 13.3 in [2], we may assume that $\mathcal{N}=\left\{N_{t}: t \in[0,1]\right\}$. Let $\mathcal{K}=L^{2}([0,1], m)$ and $\mathcal{V}=\left\{V_{t}: t \in[0,1]\right\}$, where $m$ denotes the Lebesgue measure on $[0,1]$ and $V_{t}=L^{2}([0, t], m)$. We know that $\mathcal{V}$ is a continuous nest of multiplicity one. Then by Theorem 13.10 in 2], there is an invertible operator $S$ such that $V_{t}=S N_{t}$ for all $t \in[0,1]$. If $S$ does not have any left partial factorization relative to $\mathcal{N}$, the proof is complete. Otherwise, there is a projection $E$ in $\mathfrak{D}_{\mathcal{V}}$ such that the map $N_{t} \rightarrow E V_{t}$ is unitary implementing by Proposition 1. Then it is sufficient to prove that $E \mathcal{V}$ does not have the left partial factorization property. Note that $\mathfrak{D}_{\mathcal{V}}=\left\{L_{f}: f \in L^{\infty}([0,1])\right\}$, where $L_{f}$ is the operator of multiplication by $f$ on $L^{2}[0,1]$. Then there is a Borel subset $\Delta$ of $[0,1]$ such that $E=L_{\chi_{\Delta}}$ and $E V_{t}=L^{2}(\Delta \cap[0, t])$ for all $t$, where $\chi_{\Delta}$ is the characteristic function of $\Delta$. Since $N_{t} \rightarrow E V_{t}$ is a dimension-preserving order isomorphism, we have that $\Delta$ is dense in $[0,1]$ and for any segment $J$ of $[0,1], m(J \cap \Delta) \neq 0$.

Let $\Omega$ be an open subset of $[0,1]$ such that $\Omega \cap \Delta$ is dense in $[0,1]$ and $m(\Omega)<$ $m(\Delta)$. It is trivial that $m(J \cap \Delta \cap \Omega) \neq 0$ for any segment $J$ of $[0,1]$. Define the finite Borel measure $\mu$ on $[0,1]$ by $\mu(G)=m(G \cap \Omega \cap \Delta)$ for any Borel subset $G$ of $[0,1]$. Let $\mathcal{L}=L^{2}([0,1], \mu)$ and $\mathcal{M}=\left\{M_{t}: t \in[0,1]\right\}$, where $M_{t}=L^{2}([0, t], \mu)$. Then $\mathcal{M}$ is a continuous nest of multiplicity one. Thus the map $E V_{t} \rightarrow M_{t}$ is a dimension-preserving order isomorphism of $E \mathcal{V}$ onto $\mathcal{M}$. By Theorem 13.10 in [2] again, there is an invertible operator $T$ from $E \mathcal{K}$ onto $\mathcal{L}$ such that $M_{t}=T E V_{t}$ for all $t \in[0,1]$. We next prove that $T$ does not have any left partial factorization relative to $E \mathcal{V}$. Otherwise, by Proposition 1 , there is a projection $F \in \mathfrak{D}_{\mathcal{M}}$ so that $E V_{t} \rightarrow F M_{t}$ is unitary implementing. Let $U$ be a unitary operator from $E \mathcal{K}$ onto $F \mathcal{L}$ such that $U E V_{t}=F M_{t}$ for all $t \in[0,1]$. Then for any $\alpha, \beta \in[0,1]$ with $\alpha<\beta$, we have that $U\left(E V_{\beta} \ominus E V_{\alpha}\right)=F\left(M_{\beta} \ominus M_{\alpha}\right)$.

Now let $\Omega$ be the union of a sequence $G_{n}=\left(\alpha_{n}, \beta_{n}\right)$ of disjoint relative open intervals in [0,1], and let $E_{n}=E V_{\beta_{n}} \ominus E V_{\alpha_{n}}$ and $F_{n}=M_{\beta_{n}} \ominus M_{\alpha_{n}}$ for all $n$. Then we have $\sum_{n} P_{F_{n}}=I$, and then $F\left(\sum_{n} P_{F_{n}}\right)=F$. It now follows that $\sum_{n} P_{E_{n}}=I$ on $E \mathcal{K}$ since $U$ is unitary. However, we have

$$
\begin{aligned}
\sum_{n} \oplus E_{n} & =\sum_{n} \oplus \chi_{\left(\left(\alpha_{n}, \beta_{n}\right) \cap \Delta\right)} L^{2}[0,1] \\
& =\chi_{(\Omega \cap \Delta)} L^{2}[0,1] \varsubsetneqq \chi_{\Delta} L^{2}[0,1]=E \mathcal{K}
\end{aligned}
$$

since $m(\Omega)<m(\Delta)$. This is a contradiction. Thus $T$ does not have any left partial factorization relative to $E \mathcal{V}$. The proof is complete.

Theorem 1. Let $\mathcal{N}$ be a nest. Then $\mathcal{N}$ has the left partial factorization property if and only if $\mathcal{N}$ is atomic. Moreover, if $\mathcal{N}$ is not atomic, then for each $\varepsilon>0$, there is an invertible operator $T=I+K$ where $K$ is compact and $\|K\|<\varepsilon$ such that $T$ does not have any left partial factorization relative to $\mathcal{N}$.

Proof. If $\mathcal{N}$ is atomic, then we know that $\mathcal{N}$ is injective in the sense that there exists a faithful normal expectation from $\mathcal{B}(\mathcal{H})$ onto the diagonal $\mathfrak{D}_{\mathcal{N}}$. By Corollary 5.7 in [8], we know that $\mathcal{N}$ has the left partial factorization property. 
Conversely, let $\mathcal{N}$ have the left partial factorization property. Let $G=\vee\{N \ominus$ $\left.N_{-}: N \in \mathcal{N}\right\}$. Then we know that $P_{G}$ is a core projection in $\mathfrak{E}_{\mathcal{N}}$ and $P_{G} \mathcal{N}$ is atomic while $P_{G^{\perp}} \mathcal{N}$ is continuous. We next prove that $G=\mathcal{H}$. Otherwise, we have that $P_{G^{\perp}} \mathcal{N}$ is a continuous nest on $G^{\perp}$. By Lemma 1, there is an invertible operator $S$ on $G^{\perp}$ so that $S$ does not have any left partial factorization relative to $P_{G^{\perp}} \mathcal{N}$. Let $T=P_{G} \oplus S$; then $T$ is invertible. We prove that $T$ does not have any left partial factorization relative to $\mathcal{N}$. Suppose that there is a projection $E$ in the diagonal $\mathfrak{D}_{T \mathcal{N}}$ such that $N \rightarrow E(T N)$ is unitary implementing and $U$ is a unitary operator from $\mathcal{H}$ onto $E \mathcal{H}$ such that $U N=E(T N)$ for all $N \in \mathcal{N}$. Let $F=N \ominus N_{-}$be an atom of $\mathcal{N}$. We have $U F=U\left(N \ominus N_{-}\right)=U N \ominus U N_{-}=$ $E(T N) \ominus E\left(T N_{-}\right)=E\left(T N \ominus T N_{-}\right)$. It is easy to see that $T N \ominus T N_{-}=P_{G}(N \ominus$ $\left.N_{-}\right)=N-N_{-}=T N \ominus(T N)_{-}$. Thus $G=\vee\left\{T N \ominus(T N)_{-}: N \in \mathcal{N}\right\}$ is the atomic part of $T \mathcal{N}$. It follows that $P_{G}$ is also a core projection of $T \mathcal{N}$, and then $E P_{G}=P_{G} E$. We note that $U$ is unitary. $U$ maps an atom of $\mathcal{N}$ to an atom of $E(T \mathcal{N})$. Then if $F$ is an atom of $\mathcal{N}, U F$ is an atom of $E(T \mathcal{N})$. That is, $U F=$ $U\left(N \ominus N_{-}\right)=E\left(T N \ominus(T N)_{-}\right)=E\left(N-N_{-}\right)$. Thus $U G=E G$ and $U: G \rightarrow E G$ is unitary. Then $U: G^{\perp} \rightarrow E G^{\perp}$ is also unitary. For $N \in \mathcal{N}$, we have that $U N=U\left(P_{G}+P_{G^{\perp}}\right) N=U P_{G} N \oplus U P_{G^{\perp}} N=E(T N)=P_{G} E(T N) \oplus P_{G^{\perp}} E(T N)$. It follows that $U P_{G^{\perp}} N=E P_{G^{\perp}}(T N)=E P_{G^{\perp}} S P_{G^{\perp}} N$ for all $N \in \mathcal{N}$. Thus $E P_{G^{\perp}}$ is a projection in $\mathfrak{D}_{P_{G^{\perp}} \mathcal{N}}$ such that the map $P_{G^{\perp}} N \rightarrow\left(E P_{G^{\perp}}\right) S\left(P_{G^{\perp}} N\right)$ is unitary implementing with the unitary operator $\left.U\right|_{G^{\perp}}$. It follows that $S$ has a left partial factorization relative to $P_{G^{\perp}} \mathcal{N}$ by Proposition 1 . This is a contradiction. Then $T$ does not have any left partial factorization relative to $\mathcal{N}$. This contradicts the assumption. Then $\mathcal{N}$ is atomic.

Let $\mathcal{N}$ be not atomic and $T$ an invertible operator that does not have any left partial factorization relative to $\mathcal{N}$. Then by Theorem 13.20 in [2], there is an invertible operator $T_{0}=W(I+K)$ where $W$ is a unitary operator, $K$ is compact and $\|K\|<\varepsilon$ such that $T N=T_{0} N$ for every $N \in \mathcal{N}$. It easily follows that $I+K$ does not have any left partial factorization relative to $\mathcal{N}$. The proof is complete.

Remark 1. Theorem 1 says that the converse of Corollary 5.7 in 8 holds for nests in $\mathcal{B}(\mathcal{H})$. It may be interesting to know whether the converse holds for nests in any von Neumann algebras.

For the right partial factorization property, we will see that it is equivalent to the factorization property.

Lemma 2. Let $\mathcal{N}$ be a continuous nest. Then $\mathcal{N}$ does not have the right factorization property.

Proof. Let $\mathcal{N}=\left\{N_{t}: t \in[0,1]\right\}$, and let $E_{\mathcal{N}}$ be the spectral measure such that $N_{t}=E_{\mathcal{N}}[0, t] \mathcal{H}$ (cf. Corollary 7.6 in [2]). Let $\mu$ be a finite Borel measure on $[0,1]$ mutually absolutely continuous to $E_{\mathcal{N}}$. For example, $\mu(A)=\left(E_{\mathcal{N}}(A) x, x\right)$, where $x$ is a separable vector for $\mathfrak{E}_{\mathcal{N}}$.

If $\mathcal{N}$ has the right factorization property, then for any continuous nest $\mathcal{M}=\left\{M_{t}\right.$ : $t \in[0,1]\}$ acting on $\mathcal{K}$, letting $T$ be an invertible operator such that $M_{t}=T N_{t}$ for all $t \in[0,1]$, there is a projection $E$ in $\mathfrak{D}_{\mathcal{N}}$ and a unitary operator from $E \mathcal{H}$ onto $\mathcal{K}$ such that $U E P_{N_{t}} U^{*}=P_{M_{t}}$ for all $t \in[0,1]$ by Proposition 1 . Now let $\nu$ be a finite nonatomic Borel measure on $[0,1]$ such that $\nu[0, t]$ is strictly monotone continuous (that is, $\nu(J) \neq 0$ for any segment $J$ of $[0,1])$. Let $\mathcal{K}=L^{2}([0,1], \nu)$ 
and $M_{t}=\chi_{[0, t]} L^{2}([0,1], \nu)$ for all $t \in[0,1]$. Then we have $U E P_{N_{t}} U^{*}=P_{M_{t}}$ for all $t \in[0,1]$. It easily follows that $\nu$ is absolutely continuous with respect to $\mu$.

It is an elementary fact that there exists a finite nonzero nonatmoic Borel measure $\lambda$ on $[0,1]$ so that $\mu$ and $\lambda$ are mutually singular. For example, we can construct a monotone continuous function $f$ on $[0,1]$ with $f(0)=0$ and $f(1)=1$ such that its derivative relative to $\mu$ is 0 a.e. by a similar method as on pages $144-145$ of [10]. Let $\lambda$ be the Lebesgue-Stieltjes measure induced by $f$. Now let $\nu=m+\lambda$ on $[0,1]$. Then $\nu$ is a finite Borel measure on $[0,1]$ such that $\nu[0, t]$ is strictly monotone continuous. Thus it follows that $\nu$ is absolutely continuous with respect to $\mu$ and so is $\lambda$. This is a contradiction. Then $\mathcal{N}$ does not have the right factorization property. The proof is complete.

Theorem 2. Let $\mathcal{N}$ be a nest. The following are equivalent:

(1) $\mathcal{N}$ is countable.

(2) $\mathcal{N}$ has the factorization property.

(3) $\mathcal{N}$ has the right partial factorization property.

Proof. The equivalence of (1) and (2) was well known by [7]. That (2) implies (3) is clear.

$(3) \Longrightarrow(2)$ By Lemma 2 and a method similar to the proof of Theorem 1, we can prove that if $\mathcal{N}$ has the right partial factorization property, then it is atomic. Thus by Theorem 1, $\mathcal{N}$ has the left partial factorization property. By Proposition 4.4 in [8], we have that $\mathcal{N}$ has the factorization property. The proof is complete.

Remark 2. By Theorem 2, we know that if a nest has the right partial factorization property, it has the left one as well. However, for an invertible operator $T$ with a right partial factorization relative to a nest, it does not need to have a left partial factorization relative to the nest. For example, as in the proof of Lemma 1, put $\mathcal{K}=L^{2}([0,1], m)$ and $\mathcal{N}=\left\{N_{t}: t \in[0,1]\right\}$, where $N_{t}=L^{2}([0, t], m)$. Let $\mathcal{H}=$ $\mathcal{K} \oplus \mathcal{K}$ and $\mathcal{M}=\left\{M_{t}: t \in[0,1]\right\}$, where $M_{t}=N_{t} \oplus N_{t}, t \in[0,1]$. Put $\theta\left(M_{t}\right)=N_{t}$ for all $t \in[0,1]$. Then $\theta$ is an order isomorphism from $\mathcal{M}$ onto $\mathcal{N}$. Thus there exists an invertible operator $T \in \mathcal{B}(\mathcal{H}, \mathcal{K})$ such that $T M_{t}=N_{t}$ for all $t \in[0,1]$ by Theorem 13.10 in [2. We have that $T$ has a right partial factorization relative to $\mathcal{M}$, but it does not have a left one. In fact, put $E=I \oplus 0$, where $I$ is the identity operator on $\mathcal{K}$. Then it is clear that $E \in \mathfrak{D}_{\mathcal{M}}$ and the order isomorphism $E M_{t} \rightarrow N_{t}$ is unitary implementing. By Proposition $1, T$ has a right partial factorization relative to $\mathcal{M}$. If $T$ has a left partial factorization relative to $\mathcal{M}$, then there is a projection $E \in \mathfrak{D}_{\mathcal{N}}$ such that the order isomorphism $M_{t} \rightarrow E N_{t}$ is unitary implementing by Proposition 1. As in the proof of Lemma 1, there is a Borel subset $\Delta$ that is dense in $[0,1]$ and $m(J \cap \Delta) \neq 0$ for any segment $J$ of $[0,1]$ such that $E$ is the multiplication operator by $\chi_{\Delta}$ on $\mathcal{K}$. In this case, we have $E \mathcal{K}=L^{2}(\Delta, m)$ and $E N_{t}=\chi_{[0, t]} L^{2}(\Delta, m)=L^{2}(\Delta \cap[0, t], m)$. We easily have that $E \mathcal{N}=\left\{E N_{t}: t \in[0,1]\right\}$ is of multiplicity one. This is a contradiction since $\mathcal{M}$ is not of multiplicity one. Thus $T$ does not have any left partial factorization relative to $\mathcal{M}$. It is also known that $T^{-1}$ has a left partial factorization relative to $\mathcal{N}$, but it does not have any right one.

Recall that an invertible operator $T$ has the universal factorization property if it has a factorization relative to every nest (cf. [3, 4, 5]). Gohberg and Krein in [4, 5] showed that every positive operator of the form $\lambda I+K$, where $K$ belongs to the Mac'aev ideal has this property. Davidson and Huang in [3] proved that 
if an invertible positive operator has the universal factorization property, then it must have the form scalar plus compact. We may define a similar notion for the left (resp. right) partial factorization property. That is, we say that an invertible operator $T$ has the universal left (resp. right) partial factorization property if it has a left (resp. right) partial factorization relative to every nest. However, we have the following result.

Proposition 2. Let $T$ be an invertible operator in $\mathcal{B}(\mathcal{H})$. The following are equivalent:

(1) $T$ has the universal factorization property.

(2) $T$ has the universal left partial factorization property.

(3) $T$ has the universal right partial factorization property.

Proof. We note that $T$ and $|T|$ have the same factorization property. Without loss of generality, we may assume that $T$ is positive.

The implication from (1) to (2) is trivial.

$(2) \Longrightarrow(1)$ Let $\mathcal{N}$ be an arbitrary nest, and let $\mathcal{M}=\left\{N^{\perp}: N \in \mathcal{N}\right\}$. By the assumption (2), there is an isometry $U$ such that both $U^{*} T$ and $T^{-1} U$ are in $A l g \mathcal{M}$. It follows that both $U^{*} T^{-1}$ and $T U$ are in $A l g \mathcal{N}$, which implies that $T^{-1}$ has a left partial factorization relative to $\mathcal{N}$. Then $T^{-1}$ has the universal left partial factorization property also.

On the other hand, $T$ also has a left partial factorization relative to $T^{-1} \mathcal{N}$. Thus there is an isometry $V$ such that

$$
V^{*} T\left(T^{-1} N\right) \subseteq T^{-1} N, \quad T^{-1} V\left(T^{-1} N\right) \subseteq T^{-1} N
$$

for all $N \in \mathcal{N}$. That is, $T V^{*} N \subseteq N, V T^{-1} N \subseteq N$ for all $N \in \mathcal{N}$. It follows that $T^{-1}$ has a right factorization property relative to $\mathcal{N}$. Then by Proposition 4.4 in [8], $T^{-1}$ has a factorization relative to $\mathcal{N}$. Thus $T^{-1}$ has the universal factorization property and so does $T$.

The proof of equivalence of (1) and (3) is similar. The proof is complete.

\section{ACKNOWLEDGMENT}

Part of this work was done during the first author's visit at the Department of Mathematics, Niigata University, Japan. He is grateful for the hospitality of Professor Kichi-Suke Saito. The authors would like to thank the referees whose comments and suggestions led to an improvement in the presentation of this paper.

\section{REFERENCES}

[1] W. B. Arveson, Interpolation problems in nest algebras, J. Funct. Anal., 20(1998), 208-233. MR 52:3979

[2] K.R. Davidson, Nest Algebras, Pitman Research Notes in Math., Vol. 191, Longman, London/New York, 1988. MR 90f:47062

[3] K. R. Davidson and H. Huang, Universal factorization of positive operators, Indiana Univ. Math. J, 43(1998), 131-142. MR 95c:47019]

[4] I. C. Gohberg and M. G. Krein, Factorization of operators in Hilbert space, Acta Sci. Math. Szeged, 25(1964), 90-123; English transl., Amer. Math. Soc. Transl. (2)51(1966), 155-188. MR 29:6313

[5] I. C. Gohberg and M. G. Krein, Theory and applications of Volterra operators in Hilbert space, "Nauka", Moscow, 1967; English transl., Transl. Math. Monographs, 24, Amer. Math. Soc., Providence, RI, 1970. MR 41:9041

[6] G. Ji and K.-S. Saito, Factorization in subdiagonal algebras, J. Funct. Anal., 159(1998), 191-202. MR 99k:46106 
[7] D. R. Larson, Nest algebras and similarity transformations, Ann. Math., 121(1985), 409-427. MR 86j:47061

[8] D. R. Pitts, Factorization problems for nests: Factorization methods and characterizations of the universal factorization property, J. Funct. Anal., 79(1988), 57-90. MR 90a:46160

[9] S. C. Power, Factorization in analytic operator algebras, J. Funct. Anal., 67(1986), 413-432. MR 87k:47040

[10] W. Rudin, Real and complex analysis, Third Edition, McGraw-Hill Book Company, 1987. MR 88k:00002

College of Mathematics and Information Science, Shaanxi Normal University, Xian 710062, People's Republic of China

E-mail address: gxji@snnu.edu.cn

College of Mathematics and Information Science, Shaanxi Normal University, Xian 710062, People's Republic of China 